\title{
The Right Treatment for the Right Patient - Personalised Treatment of Breast Cancer
}

\author{
Die richtige Therapie für die richtige Patientin - \\ personalisierte Behandlung des Mammakarzinoms
}

Authors

Affiliations
A. Scharl ${ }^{1}$, T. Kühn ${ }^{2}$, T. Papathemelis ${ }^{1}$, A. Salterberg ${ }^{3}$

${ }^{1}$ Frauenklinik, Klinikum St. Marien Amberg, Amberg

${ }^{2}$ Frauenklinik, Klinikum Esslingen, Esslingen

${ }^{3}$ Department of Gynaecology and Obstetrics, Sana Clinics, Cham
Key words

- breast cancer

- lifestyle changes

- compliance

- excessive treatment

over-diagnosis

Schlüsselwörter

- Brustkrebs

- Lifestyle-Veränderungen

- Compliance

- Übertherapie

- Überdiagnostik

Deutschsprachige Zusatzinformationen online abrufbar unter: www.thieme-connect.de/ ejournals/toc/gebfra

\section{received 7.4.2015 \\ revised $\quad 4.5 .2015$ \\ accepted 19.6.2015}

\section{Bibliography}

DOI http://dx.doi.org/

10.1055/s-0035-1546270

Geburtsh Frauenheilk 2015; 75 :

683-691 @ Georg Thieme

Verlag KG Stuttgart · New York ·

ISSN 0016-5751

\section{Correspondence}

Prof. Dr. med. Anton Scharl

Klinikum St. Marien Amberg

Mariahilfbergweg 7

92224 Amberg

scharl.anton@

klinikum-amberg.de

\section{Abstract}

$\nabla$

The probability of healing breast cancer has been greatly improved in recent decades through the introduction and optimisation of multi-modal therapies and interdisciplinary treatments. Today, in addition to surgery or radiation, most patients receive a systemic treatment. To prevent excessive treatment, patients whose prognosis is so good that certain adjuvant therapies can be foregone or reduced must be identified. A lack of compliance with therapy, especially in the endocrine therapies stretching over years, is a further problem. As only treatments that are also carried out can improve chances of survival, efforts to improve compliance must be intensified. Studies show that lifestyle changes influence the efficiency of medication on the one hand, and on the other hand can also by themselves achieve a relevant improvement of the prognosis. Therefore, it is time not only to treat the tumour, but to also focus on the patient as a whole in therapeutic interventions.

To see clearly, one often only needs a change of perspective.

Antoine de Saint-Exupéry

\section{Introduction}

\section{$\nabla$}

The incidence of the disease and gender medicine have increasingly put breast cancer in the public spotlight since the end of last millennium. It has become a "political" disease. This helped to pioneer and advance the necessary developments in

\section{Zusammenfassung \\ $\nabla$}

Die Heilungswahrscheinlichkeit des Mammakarzinoms wurde in den letzten Jahrzehnten durch Einführung und Optimierung multimodaler Therapien und interdisziplinäre Behandlung stark verbessert. Heutzutage erhalten die meisten Patientinnen neben der Operation eine Bestrahlung und systemische Therapie. Um eine Übertherapie zu vermeiden müssen diejenigen Patientinnen besser identifiziert werden, die eine so gute Prognose haben, dass auf manche begleitende Therapie verzichtet oder reduziert werden kann. Ein weiteres Problem ist die oft mangelnde Therapietreue (Compliance) v.a. im Bereich der jahrelangen endokrinen Therapien. Da nur eine Behandlung, die auch durchgeführt wird, das Überleben verbessern kann, müssen die Bemühungen zur Verbesserung der Compliance intensiviert werden. Studien zeigen, dass Änderungen des Lebensstils einerseits die Wirksamkeit von Medikamenten beeinflussen und andererseits auch alleine relevante Verbesserungen der Prognose bewirken können. Daher ist es an der Zeit, nicht nur den Tumor, sondern die ganze Patientin in den Mittelpunkt der therapeutischen Interventionen $\mathrm{zu}$ stellen.

Germany. In many ways breast cancer has became a "model cancer" for the development of oncology: The introduction of population-based quality-assured screening programmes, multimodal therapies including the integration of numerous disciplines into the treatment concept, the interlinking of disciplines in breast cancer centres, certifications combined with strict structural specifications and structured quality assurance, systematic development of prevention, diagnostics and therapy through studies, the development of evidence-based S3 guidelines - all 
these structures, which are a matter of course in oncology in 2015 , started with the entity of breast cancer.

The development of therapies combined with structural and educational measures immensely improved the prognosis of breast cancer in the last decade. According to Cancer Research UK, in Great Britain the age-standardised mortality rate was almost halved within 30 years; from an all-time high of 42 deaths per 100000 women at the end of the 1980s, to 24 deaths per 100000 in 2012 [1]. Between 2000 and 2009, the mortality rate in the USA fell by $1.9 \%$ per annum. The SEER Registry data currently show a relative five-year rate of survival in the Stages 0 and I of $100 \%$, in Stage II of $93 \%$, and for all Stages 0-IV a total of $89 \%[2]$.

In Germany too the mortality rate for breast cancer is on the decline. The mortality rate for $40-45$-year-old patients fell by $33 \%$ between 1990 and 2000 [3]. According to information of the Robert Koch Institute, mortality is expected to fall by a little over $9 \%$ for the five-year period between 2007 and 2012, which is in line with the trend in the EU. In 2010, the mortality rate in Germany was at 16.2 per 100000 women [4]. The relative five-year survival rate for all tumour stages was $86 \%$ in Germany in 2008 [3].

The success story was achieved through the consistent expansion of adjuvant therapies. As a result, multiple adjuvant treatments are recommended today for almost all patients with invasive breast cancer or DCIS.

Despite the satisfaction about the high cure rates that have been achieved, three serious problems remain:

In spite of all our therapies, women are still dying from breast cancer. Justifiably, the search for new therapy options is a focus of research.

The second problem is just as important; however, it receives far less attention: We also subject patients that could probably have been cured by surgery alone to adjuvant measures with many side effects. Therefore, we need to better identify patients whose prognosis is so good that certain adjuvant therapies can be foregone and the treatment de-escalated. A gradual reduction of the intensity of therapy has been carried out successfully for breast and axilla surgery in the last decades - fewer operations while maintaining equal oncological safety.

There are fewer approaches for the de-escalation of treatment in the field of adjuvant systemic and radio-oncology therapies, although this appears to be increasingly important. The mammography screening programme achieves a more frequent diagnosis of early tumour states or precursor lesions. This inevitably results in over-diagnoses, i.e. the discovery of cancers which would not have resulted in clinical manifestation in a woman's lifespan [5]. Subjecting these patients to excessive treatment is doubly fatal.

A lack of compliance, especially in the endocrine therapies stretching over years, is a further problem. Only treatments that are also carried out can improve the chances of survival. We spend much energy on the indication for adjuvant chemotherapy and radiation and on convincing patients of the necessity for it. Following the acute phase of treatment, the attention with regard to maintaining a necessary long-term therapy strongly decreases, on an individual, structural and institutional level. For each diagnostic and therapeutic measure, one has to clearly and comprehensibly point out to the patient the possible benefits compared to the possible risks. On this basis, the patient can be convinced of the necessity of the measure and support it as her own personal decision.
It is time to investigate whether and for which patients less therapy is possible without compromising the healing of the disease. Measures that do not result in a (relevant) improvement of the prognosis should therefore not be taken. On the other hand, efforts need to be made to improve the patients' acceptance of efficient and necessary therapies.

Good treatment must not only take into account the facts of the tumour, but also the characteristics, expectations and habits of the "host". Only then is the treatment right for the right patient.

\section{Sometimes Less is More \\ $\nabla$}

A lot does not always achieve much. The development of surgical therapy for breast cancer from the Rotter-Halsted radical surgery to breast-conserving therapy (BET) has shown that less can indeed be more.

In diagnostics and therapy, some habits, recommendations, "logic" and "certainty" must continue to be questioned based on the existing available data.

Based on retrospective data, in the surgical therapy of DCIS until a few years ago a tumour-free resection margin of $1 \mathrm{~cm}$ was demanded. Many follow-up resections and mastectomies were performed to achieve this. Since then it has been shown that also a more narrow resection margin of a minimum of $2 \mathrm{~mm}$ does not carry an increased oncological risk if accompanied by radiation therapy. [6-9].

For the surgical therapy of invasive cancer a "no tumour on ink" tumour-free resection margin is sufficient; the S3 Guideline demands $1 \mathrm{~mm}[7,8,10]$. The reality of surgical procedures shows, however, that the idea that a more generous surgical therapy positively influences the patient's destiny has not yet been abandoned. Therefore, wide excisions are performed with plastic reconstruction or even mastectomies of the affected breast, even if breast-conserving therapy would have been possible. In the USA this development has resulted in fewer patients receiving breastconserving therapy than mastectomies [11]. Fortunately, such a tendency is currently not the case in Europe [12]. In the USA as well, additional prophylactic mastectomies of the other breast are being performed in women with unilateral breast cancer and without BRCA mutations. In California, twice as many unilateral or bilateral mastectomies are being performed on women under the age of 40 than breast-conserving surgeries [11]. Does this aggressive surgical therapy offer an oncological benefit?

A recent publication by Pilewskie and colleagues [13] shows that even in aggressive and triple-negative tumours with unfavourable prognosis, a more generous resection does not result in a lower local recurrence rate compared to "no tumour on ink". The higher local recurrence rate of these tumours is due to the aggressive tumour biology, which not even more extensive surgery can overcome. A joint recommendation of American Medical Societies based on a systematic literature research of 33 studies involving more than 28000 patients found that an unfavourable tumour biology does not justify a larger resection: wide resection margins do not significantly reduce the risk of local recurrence, irrespective of the biology of the tumour, the age of the patient, lobular carcinomas or intraductal tumour parts. However, resection margins affected by tumour are associated with twice the rate of risk of local recurrence compared to an R0 resection. This heightened risk is not reduced by favourable tumour biology, radiation therapy or systemic treatment [14]. Compliance with this 
recommendation can reduce the numbers of re-operations, lower mortality rates, improve the cosmetic result and reduce costs. Using the California Cancer Register, Kurian and colleagues [11] investigated the frequency and the results of different surgical procedures (unilateral mastectomy, breast-conserving surgery including radiation, bilateral mastectomy) in women with unilateral breast carcinomas. From 1998 to 2001, the incidence of bilateral mastectomies increased annually by $14 \%$, from 2.0 to $12.3 \%$; in women under the age of 40 years, from 3.6 to $33 \%$. Compared to breast-conserving therapy, there was no significant difference in the 10-year overall mortality of bilateral mastectomies (16.8 vs. $18.8 \%$ ). For unilateral mastectomies, however, the mortality was significantly higher (16.8 vs. $20.1 \%$ ). In accordance with other publications [15], the authors could find no evidence showing that a prophylactic mastectomy of the other breast in breast cancer patients without a predisposing genetic mutation for breast cancer has any benefit for survival; it is therefore oncologically meaningless.

Diagnosticians and therapists often fall victim to the misconception that detection of a prevalent cancer always works in the patient's favour, as an early diagnosis increases the chance of cure. Patients too often believe this themselves. With the introduction of mammography screenings, the issue of over-diagnoses, i.e. the evidence of biologically irrelevant cancers, became evident [5]. However, over-diagnoses can also occur for cancers that would lead to death if left untreated. Here the irrational use of radiological diagnostics must be noted. The following example explains this.

The concept of breast-conserving therapy was developed 30 years ago, when magnetic resonance imaging of the breast did not yet play any role. Countless studies proved that the cure rate in breast-conserving therapy is identical to that of a mastectomy [16]. As we have now learnt from modern diagnostics including MRIs, in addition to the tumour that resulted in the diagnosis of cancer there are quite often smaller tumours present in the breast that were formerly overlooked and therefore not excised - with no negative effect on the recurrence and cure rate. Against this background, one should be sceptical of the hope that a broad use of magnetic resonance imaging in breast cancer that has already been diagnosed will improve the prognosis. Indeed MRIs detect multi-focal and multi-centric lesions which would not have been noticed without this technology. But do patients really benefit from this?

In two meta-analyses, Houssami and colleagues showed that a routinely performed, preoperative MRI brings no clinical benefit and can even be disadvantageous for patients. One meta-analysis was comprised of nine studies (two randomised, seven cohort studies) with 3112 patients, for whom the surgical therapy with and without routine preoperative MRIs was compared. In the group of patients with MRI diagnostics, the rate of initial mastectomies was twice the rate of those without MRIs (16.4 vs. $8.1 \%$ ), without the incidence of follow-up resections being reduced (11.6 vs. $11.4 \%$ ). The rate of conversions from breast conservation vs. mastectomy was also equal. In conclusion, almost 50\% more mastectomies were performed on the group of women with MRIs than on the group without MRIs (25.5 vs. 18.2\%). In lobular carcinomas, MRIs did indeed slightly reduce the re-excision rate, at the cost however of more frequent mastectomies, which means that there was no clear clinical benefit from MRIs also in this group [17]. The second meta-analysis of four studies involving 3169 patients showed that a preoperative MRI of the breast did not reduce the recurrence rate: After an observation period of eight years, neither the frequency of local recurrence nor of distant metastases in the groups with and without MRIs differed [18]. Therefore, a MRI should not be performed routinely but only when a specific issue needs to be clarified.

The presence of metastases turns a potentially curable disease into an incurable one, and changes the treatment goals. The routine search for metastases in lungs, liver and bones has no evidenced benefit in patients with a tumour with a low potential for metastasis (stage 1 and 2) who are experiencing no symptoms, but is potentially harmful. A recently published meta-analysis confirmed that in stages 1 and 2 the prevalence of metastases is low (0.2 resp. 1.2\%) [19]. This is significantly lower than the rate of false positive findings in modern imaging $[19,20]$. The rarer the occurrence of metastases, the higher the proportion of patients who suffer additional morbidity from fear, unnecessary radiation, additional (invasive) diagnostics and excessive treatment as a result of dubious or false positive findings. The American Society of Clinical Oncology (ASCO) has identified not searching for metastases by PET, CT and bone scintigraphy in stage 1 and stage 2 breast cancer patients free of symptoms as one of five key measures for improving oncological treatment [21]. However, a current publication shows that this recommendation has not resulted in a reduction of staging measures [22]. M-staging is meaningful in symptom-free patients prior to (neo-)adjuvant chemotherapy, as the aggressive (neo-)adjuvant poly-chemotherapy regimes are not indicated for metastasised patients.

\section{Therapy Compliance}

Even the best therapy is only effective if it is being carried out. Therefore, optimal compliance is of crucial importance. It can make more sense to perform a limited range of therapy modalities with optimal compliance than to carry out many halfhearted measures.

Experience has shown that therapy compliance is lower the longer the therapy is to run and the more distant the contact with the treating doctors. This is why endocrine therapy, which must be carried out over many years, and in which the medical doctor is only visited a few times a year, is particularly susceptible to a lack of therapy compliance.

A five-year endocrine therapy improves the 15-year survival chances of patients with receptor-positive breast cancer by one third [23]. Only half of the women with breast cancer, however, undergo this treatment over the recommended five years [24, 25 ]. This lack of compliance is associated with a significantly increased risk of death. At less than $60 \%$ therapy compliance, the mortality risk is nearly four times higher than in good therapy adherence [25].

Side-effects are a common reason for ending treatments. Muscle and joint aches and menopausal symptoms are a typical consequence of endocrine therapy, but also typical complaints associated with ageing. The causality requirement causes new symptoms to be perceived as a consequence of the treatment, even if they are actually unrelated. A current study shows that patients are likely to stop endocrine treatment prematurely if prior to the start of their therapy they experience (menopausal) symptoms typical of endocrine treatment (sleep disorders, fatigue, mood disorders, anxiety, problems concentrating). If three to five such symptoms were present prior to the start of the treatment, the probability of stopping an aromatase inhibitor treatment was twice as high as in women with two such symptoms at most [26]. 
On the other hand, patients and the treating doctors have many effective possibilities available to improve hormone-related symptoms with medication, but also with lifestyle measures such as sport, mind-body medicine, weight loss or acupuncture $[7,8]$. One current study proves for example that structured physical activity programmes can relieve arthralgia caused by aromatase inhibitors [27].

Unfortunately, symptoms caused by hormone treatments are not sufficiently discussed in the doctor-patient communication. Comparative studies show that patients experience side-effects far more often and more severely than doctors perceive [28]. This means they are not addressed and treated adequately in the doctor-patient interaction.

In the IBIS II Breast Cancer Prevention Study a five-year treatment with anastrazole was tested in comparison with a placebo. The compliance over five years in the placebo group was $72 \%$; in the anastrazole group it was only slightly lower, at 68\%: more than two-thirds of the healthy women continued treatment over five years and accepted the side-effects [29].

The significantly better compliance in the prevention study compared to everyday treatment showed what the provision of detailed information, motivation, persuasion and close support can effect [29]. It is important to convince patients of the necessity of the treatment, increase the awareness of side-effects and of symptoms unrelated to the treatment through careful anamnesis, and lastly to adequately treat these symptoms. If therapy compliance can be increased with these measures, more lives can possibly be saved than with additional chemotherapy.

The data from the SOFT and TEXT study must be viewed critically against this background. These randomised phase-three studies tested in pre-menopausal patients a five-year endocrine treatment with either tamoxifen alone or the combination of tamoxifen or exemestane with ovarian suppression (OFS). The studies showed benefits of OFS in patients that had such high risks that chemotherapy had been indicated and whose ovarian function had recovered following chemotherapy. Here, the aromatase inhibitor exemestane in combination with OFS showed better results than tamoxifen. The benefits consisted of better recurrence-free and breast-cancer-free survival, whereas the overall survival in the observation time of an average of 67 months did not differ significantly. The combination therapy had significantly more side-effects than the mono-therapy with anti-oestrogen $[30,31]$. When the side-effects profile results in increased treatment terminations, the hoped-for positive effect can quickly turn into a negative one.

\section{Over-Treatment and Under-Treatment Through Adjuvant Chemotherapy \\ $\nabla$}

Data of the EBCTCG meta-analysis of randomised studies suggest that systemic adjuvant chemotherapy lowers the mortality of breast carcinomas by a third, irrespective of other variables such as age, TN status or hormone receptor expression [32]. Without chemotherapy the 10-year mortality was at just under $36 \%$, while with poly-chemotherapy using anthracyclines and taxanes mortality was at $21 \%$. This means that in $21 \%$ of patients, insufficient or improper treatments were performed, since despite having undergone chemotherapy they did die. For this group, innovative therapy approaches are urgently required.

According to the meta-analysis data [32], 64\% of patients in the chemotherapy-free groups of the randomised studies survived
10 years. This suggests that about this percentage of patients in the groups with chemotherapy would have survived for 10 years even without this treatment. Consequently, approximately $64 \%$ of the women in the chemotherapy groups received excessive treatment. To reduce unnecessary morbidity associated with treatment, these patients should be identified by determining the individual oncological risk and the benefit of chemotherapy as precise as possible. Therapeutic successes from studies always refer to a collective and show relative improvements. For the individual patient, however, the absolute improvement is what is crucial. This depends on the individual risk. A low absolute risk means a low absolute gain from therapy ( Fig. 1). Unfortunately, to date we have only limited possibilities of identifying those patients who need chemotherapy and for whom it also works. From the combination of the mortality risk of the individual patient and the relative benefit of chemotherapy determined in the studies, a risk/benefit ratio is calculated. This involves prognostic and predictive factors such as oestrogen and HER2/new-status, TN status, proliferation rate and in some cases also gen expression analyses which reflect the biology of a tumour, i.e. its natural progression (prognosis) or the probability of its response to therapy (prediction) $[7,8,16]$.

However, the issue is not only the decision whether chemotherapy is indicated, but also the selection of the regime. $15 \%$ of the patients in the EBCTCG meta-analysis survived because of the anthracycline-based and taxane-based treatment [32]. This combination was more effective than only an anthracycline-based treatment. Consequently, such a combination therapy is usually deployed. However, with an increasingly lower risk, the absolute gain through this chemotherapy is also lower and the advantage over a treatment that uses just one of the two substances is smaller. To improve the balance of advantages and disadvantages, there should be identified patients whose prognosis is already so favourable that the combination can be omitted without compromising the probability of recovery. Studies on such a de-escalation of therapy are the exception.

A study on HER-2/new-positive breast cancer patients [33] shows that this path is possible. The combination of anthracyclinebased and taxane-based or platinum-based and taxane-based poly-chemotherapy with trastuzumab reduced the risk of recurrence of this tumour to $19 \%$ [16]. In a single-arm study, Tolaney and her colleagues posed the question whether there is a patient collective for whom a sole mono-therapy with a taxane (parallel to the anti-HER-2 targeted therapy) would be possible without deterioration of the prognosis. 406 patients with a nodal-negative, HER-2/new positive tumour smaller than $3 \mathrm{~cm}$ in diameter underwent mono-chemotherapy with 12 weekly cycles of paclitaxel plus trastuzumab for one year. The prognosis was excellent. After three years the recurrence-free survival rate was $99 \%$. In the 12 recurrences, only two cases of distant metastases were detected. [33]. Therefore, a de-escalation of poly-chemotherapy seems quite possible in selected collectives.

The better the efficiency of a therapy in a collective can be predicted, the lower the proportion of patients who do not benefit from the treatment and the more precise the targeting of the medical indication. Neo-adjuvant chemotherapy studies proved that a platinum-based chemotherapy of triple-negative breast cancer can be highly effective. However, this is also associated with a high toxicity. It is therefore very important to identify patients that will actually benefit from the administration of platinum. Translational research projects in this collective showed that the effect increased with an increasing number of tumour- 


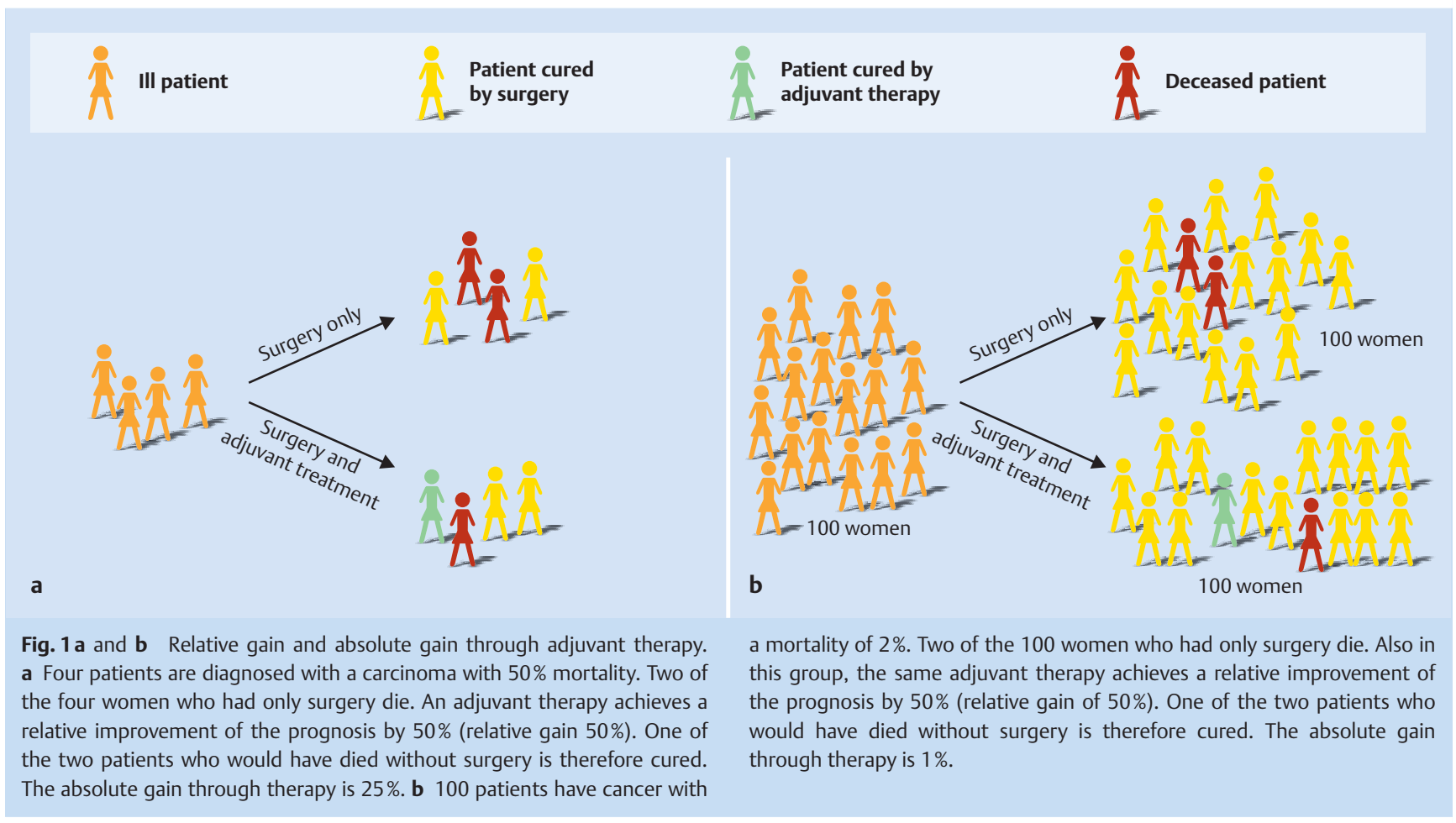

infiltrating lymphocytes [34]. However, it was also demonstrated that the high level of platinum-sensitivity is apparently limited to triple-negative tumours in women with a positive family history or with BRCA mutations [35]. This shows the clinical importance of the development of new predictive markers, which can help avoid unnecessary toxicity caused by applying ineffective therapies.

\section{Treatment Goal - Reducing Mortality or Reducing Recurrence? \\ $\nabla$}

Treatment of early breast cancer follows a curative intention. Healing the patient is the goal. The efficiency of a treatment is mainly measured by the reduction of mortality. A second treatment goal is to reduce the loco-regional or distant recurrence rate. Whereas distant metastases in women with longer life expectancy impacts on the mortality due to incurability, a local recurrence is initially a curable event. It causes physical and psychological morbidity but does not necessarily limit the lifetime of the patient. The reduction of mortality and the recurrence rate must therefore be strictly differentiated in their therapeutic value. This applies in particular to older patients with a limited natural life expectancy. When medically indicating the treatment and in patient communication it must be made clear whether an intervention will lower the mortality or "only" the risk of recurrence, without affecting survival.

This distinction is particularly relevant in breast radiation following a breast-conserving operation (BEO) of a DCIS. DCIS is a precursor to cancer which will only in some cases become an invasive cancer. Unfortunately, we do not know for sure how high this proportion is $(30,50$ or $70 \%$ ?), nor can we predict in individual cases whether such a progression will occur [6]. All these women are recommended to have the lesion removed completely, and to undergo radiotherapy as well following a breast-conserving op- eration. According to the current meta-analysis of randomised studies by the EBCTCG [36], this reduces the 10-year risk of an ipsilateral local recurrence by more than half to approximately $13 \%$. The relative risk reduction also applies to smaller lesions and tumour-free resection margins. It is independent of age and endocrine therapy. No sub-collective that did not benefit from radiation therapy could be defined. However, the absolute gain for patients depends on the individual risk of recurrence. Radiation therapy had no impact on the mortality. Neither the breast-cancer related mortality, nor death due to a different cause or the overall mortality is changed by radiation therapy; the survival rates for DCIS are excellent and are almost equal to those of a normal collective without breast disease [37].

In mammography screening the proportion of DCIS in all breast cancer cases has now reached $20 \%$ [38]. Every fifth "breast cancer patient" therefore is diagnosed with a pre-cursor of cancer, only a proportion of which will advance to an invasive cancer. This means that a relevant proportion of these women are treated due to a diagnosis that would never have made them ill and that they most probably would never have become aware of. Surgical removal of the lesion alone can control DCIS in such a way that the patient's life is not shortened. Under these conditions, is it justified that expert associations, guidelines and certification specifications demand radiation therapy for the majority of these women? Would it not rather be more appropriate to inform the patients that across all risk groups about seven patients need to undergo radiation therapy to prevent a local recurrence in one of them and that about 14 patients would need to undergo radiation therapy in order to prevent one invasive cancer in one of them $[36,37]$ ? Should one not explain that the mortality of the disease is also virtually nil without radiation therapy? The patient, without fearing for her life, could then decide whether the effect of radiation is worth the stress.

In breast-conserving operations of invasive cancer the radiation of the breast also reduces the local recurrence rate. Unlike in 
DCIS, it does however improve the survival rate: in a meta-analysis by EBCTCG with a follow-up observation period of 15 years, radiation therapy halved the annual recurrence rate and lowered the annual breast cancer mortality by a sixth. In different risk collectives the relative risk reduction remains the same; the absolute gain, however, falls with lower risk [39]. For clarification purposes, nodal-negative patients were divided into three risk groups for which $\mathrm{a} \geq 20 \%, 10-20 \%$ and $<10 \%$ absolute reduction of the 10 -year risk of recurrence was predicted. The absolute reduction of the 15 -year mortality in these groups was $7.8 \%$ in the high risk group, $1.1 \%$ in the medium risk group and $0.1 \%$ in the low risk group. For nodal-positive patients the mortality reduction after 15 years was $8.5 \%$.

\section{Adjuvant Treatment and Age}

With increasing age, the remaining lifetime is reduced. As trivial as this statement sounds, this must be considered in the treatment plans of older patients. The best adjuvant therapy can only prevent cancer-related death, but it cannot increase the natural life expectancy.

Schonberg and colleagues [40] examined the influence of the diagnosis of breast cancer on the survival of older women by comparing the survival rates of 100,000 breast cancer patients over the age of 67 years to those of women without breast cancer, based on the USA SEER data (Surveillance, Epidemiology and End Results) in a matched-pair analysis. During a median observation period of 7.7 years, women with DCIS or stage 1 breast cancer had a 30 or $20 \%$ better survival than the control groups without breast cancer. The mortality of breast cancer patients was only higher from stage 2 . The older the women were, the lower the impact of the breast cancer diagnosis on mortality, irrespective of the stage [40].

With adequate treatment the prognosis quoad vitam in women with early breast cancer diagnosed at the end of the seventh decade of life is therefore excellent. One question that arises is what consequences a reduction of therapies has at this age.

Two randomised studies examined the impact the foregoing of radiation after a breast-conserving operation has on the recurrence rate and mortality in older women with nodal-negative and receptor-positive tumours and endocrine therapy. Hughes and colleagues [41] randomised women over the age of 70 years with tumours up to $2 \mathrm{~cm}$, and Kunkler et al. [42] women over the age of 65 years with tumours up to a diameter of $3 \mathrm{~cm}$ into one group with radiation therapy and one group without radiation therapy. In both trials the local recurrence rate was significantly higher without radiation therapy. However, in both groups the mortality did not differ. In Hughes' study $98 \%$ of patients with and $90 \%$ of patients without radiation were recurrence-free after 10 years, while the 10-year survival rate was 67 and 66\%. 319 women had to undergo radiation to prevent 21 local recurrences, corresponding to a "number needed to treat" of 15 . Breast cancer was the cause of death in only $6 \%$ of the deceased women [41]. In the study by Kunkler et al., the recurrence rate was $1.3 \%$ after five years when radiation was performed, compared to $4.1 \%$ without radiation. The five-year overall survival rate was $94 \%$ in both groups [42].

Accordingly, breast radiation therapy also significantly reduces the recurrence rate following breast-conserving therapy in older patients; however, given adequate endocrine treatment and negative nodal status this does not result, at least within 10 years, in an improved survival probability; few women of this age with tumours with a favourable prognosis die of breast cancer.

Therefore one can offer women who match the studied collective the option to forego radiation therapy, provided they undergo consistent endocrine treatment - at the cost, however, of a higher recurrence rate of about $4 \%$ after five years and about $10 \%$ after 10 years. However, the calculated life age should not be used as a basis for these considerations, but rather the life expectancy. The question whether mono-chemotherapy improves the prognosis in older women was examined in the prospective randomised ICE study [43]. Women over the age of 65 years with breast cancer and a higher risk ( $\mathrm{N}+$ or $\mathrm{T}>2 \mathrm{~cm}$ or $\mathrm{G} 2-3$ or hormone receptor-negative) received six cycles of mono-chemotherapy with capecitabine plus biphosphonate (ibandronate) for two years, or only the biphosphonate. All patients with receptor-positive tumours received endocrine treatment. After an observation period of 10 years, the recurrence rate and the overall survival rate were identical in both collectives. One conclusion of this is that "mild" chemotherapy has no effect and that, with indication, anthracyclines or taxanes should be given as the guidelines suggest for younger women.

Furthermore, one can draw more conclusions from this study. The average age of patients was 71 years; half of these were nodal-positive, while a third had G3 tumours. The five-year overall survival was $90 \%$ and the 10 -year survival rate was $60 \%$. According to the calculation by adjuvantonline [44], the 10-year survival rate for a 71-year-old patient of age-appropriate health without breast cancer is $73 \%$. With optimum anthracycline- and taxanebased chemotherapy and endocrine treatment, a nodal-positive, receptor-positive patient with a G2 tumour smaller than $3 \mathrm{~cm}$ has a 10 -year survival rate of $61 \%$. A nodal-negative, receptorpositive patient with a G3 tumour of a diameter of $2-3 \mathrm{~cm}$ has a survival rate of $65 \%$; with endocrine therapy only, these rates are estimated to be 54 and $61 \%$. This rough estimation does not allow for an exact statement on the effect of the adjuvant chemotherapy given in the study but does suggest that with optimal chemotherapy the 10-year survival rate would have increased only slightly in the examined collective. Then again, the prognosis in the ICE study was more favourable than would have been expected for a pure endocrine treatment. The difference in the prognosis may be explained by the benefit from adjuvant biphosphonate treatment (see the following section).

\section{Bisphosphonates as a Supplement to Adjuvant Options $\nabla$}

Nearly two decades ago Diehl and colleagues [45] provided the first evidence for the efficiency of bisphosphonates in the adjuvant treatment of breast cancer with regard to the reduction of distant metastases. In a new meta-analysis based on individual patient data from randomised studies, adjuvant treatment with bisphosphonates over several years resulted in a significant reduction of bone metastasis in post-menopausal women and an increase of the overall survival after 10 years by a (relative) $17 \%$, and an absolute of about $2 \%$. No positive effect was detected in pre-menopausal women [46]. Although not all randomised studies could demonstrate the adjuvant efficiency of bisphosphonates, especially those which did not consider the menopause status, there is little doubt about the positive effect. As bisphosphonates also counteract the risk of osteoporosis, which could be a consequence of an anti-tumour treatment, they are a good option for improving the prognosis of post-menopausal breast can- 
cer patients. Above all, bisphosphonates are an additional treatment offer. The downside is that there is no approval for adjuvant anti-tumour treatment.

\section{Cancer is also Part of the Body \\ $\nabla$}

The focus of cancer therapy was and still is on the tumour itself. However, the cancer is a part of the patient's body; although it is out of control, it is still subject to the laws of biology and physiology, depends on nourishment and various metabolism regulation by the body, and it must find a balance with the body's immune system. Treatment of cancer also takes place in the patient's body; the effect is therefore subject to various metabolic modifications.

In recent years these banal facts have gained more attention. The influence of sports, nutrition, metabolism and obesity on carcinogenesis, on the efficiency of oncological treatments and on the prognosis of the patient are the subject of large-scale prospective clinical trials [47]. The findings of previous studies are still contradictory and have not yet uncovered the relationships. However, some certainties are becoming clear. Sport and exercise not only reduce the side-effects of therapies and fatigue; observational studies indicate that the incidence and prognosis of breast cancer is also improved $[7,8,48,49]$. Malnutrition and obesity can reduce the effects of endocrine therapies such as the efficiency of aromatase inhibitors, and may also have a direct impact on tumour growth and prognosis [47-49].

The randomised WINS Study (Women's Intervention Nutrition Study) started at the beginning of 1994 and examined the influence of nutrition on prognosis in almost 2500 women aged between 48 and 79 years with early breast cancer. All patients received standard oncology treatment. In the intervention group, intensive support started within six months following diagnosis and continued for five years to reduce the intake of calories through fat to below $15 \%$; the control group only received general nutritional recommendations. Interim results already showed a moderate loss of weight and a $24 \%$ lower recurrence rate in the intervention group [50]. After an observation period of 15 years, the overall mortality in the intervention group was also lower; however, the difference was not statistically significant (13.6 vs. $17 \%$ ). Remarkably, a significantly lower overall mortality was recorded in women with receptor-negative tumours, 36\% lower in oestrogen receptor-negative cancers and $56 \%$ lower in oestrogen (ER) and progesterone receptor-negative (PR) cancers. After 10 years the proportion of the surviving patients with ER-negative tumours was 90 versus 65\%, and in ER and PR-negative tumours 92 versus 65\%. The average survival in the control arm was 11.7 years; in the intervention group this was extended to 13.6 years in women with ER-negative cancers and to 14.0 years in women with ER and PR-negative tumours. The HER2/new status of the patients is not known. Statistically, however, about three-quarters of the hormone receptor-negative cancers should be triple-negative [51]. Despite all limitations of the study, the prospect that a change in nutrition and weight loss could improve the survival chances of this group of patients with a very unfavourable prognosis remains extraordinary.

These preliminary findings emphasise that we are making it too easy for ourselves and for the patients when we reduce breast cancer therapy to the application of a cocktail of surgery, radiation, chemotherapy and endocrine therapy. An optimal therapeutic success rather requires intensive support and counselling of the patient to enable her to change her lifestyle sustainably and for the rest of her life towards healthier nutrition, sufficient exercise and a normal weight.

So far, this necessity is not sufficiently represented in the guidelines, certification criteria and compensation systems. They strictly and unilaterally demand, monitor and fund the application of "steel, radiation and chemistry" according to rigid rules. Doctors and clinics handle this via a check-list and the medical funds compensate them for exactly these services. The implementation of the finding that lifestyle interventions may effect improved prognoses in the same magnitude as the demanded conventional adjuvant therapy modalities is also still in its infancy. Nutritional counselling and exercise programmes are not an integral part of the treatment and care concepts of breast cancer patients, for neither the service providers nor the cost carriers.

The main task for the future is to understand the disease of "breast cancer" so well that we can recognise, with a high level of certainty, whether individual patients can be cured by surgical therapy alone, or if they would benefit from additional, adjuvant treatments, and if so, which ones. To do this, the therapy goals of survival and "no recurrence" must be considered separately, and the age and remaining life expectancy of the patients must be taken into account. Therapy concepts are effective only when the patient understands them and accepts the side-effects. Combined with a close relationship with the treating doctor, who records and treats the side-effects, this ensures a high level of compliance, which is crucial for the success of the treatment. Diagnostic measures also require a special indication and targeted questioning. Diagnostics are not harmful per se, but carry the risk of false positive findings, resulting in patients being subjected to additional radiation exposure, psychological stress and the risk of surgery for invasive diagnostic measures, for the purposes of further clarification. Modern multi-modal treatment concepts must not only include the "hard" biological factors of the tumour; they must also include the patient as a therapeutic goal and recognise her expectations, attitudes and lifestyle and steer these into a therapeutically favourable direction. A lot remains to be done to apply the right therapy to the individual patient and not only to the tumour.

\section{Conflict of Interest \\ $\nabla$}

None of the authors has conflicts of interest regarding the content of the manuscript.

The author A. Scharl received fees from the following companies: Celgene, Roche, Novartis, TEVA, Amgen, AstraZeneca, Sanofi, Glaxo, Eisai, Riemser.

The author T. Kühn received fees from the following companies: Novartis, Genomic Health, Hoffmann-La Roche.

\section{References}

1 Cancerresearch UK. Age-standardised mortality rates, females, UK, 1971-2012. Online: http://www.cancerresearchuk.org/cancer-info/ cancerstats/types/breast/mortality/uk-breast-cancer-mortalitystatistics\#trends; last access: 02.03.2015

2 SEER Databank. Online: http://seer.cancer.gov/statfacts/html/breast. html; last access: 02.03.2015

3 Robert Koch-Institut. Gesundheitsberichterstattung des Bundes, Heft 25, Brustkrebs. 2012. Online: http://www.rki.de/Krebs/DE/Content/ Publikationen/Krebs_in_Deutschland/kid_2012/kid_2012_c50.pdf? _blob=publicationFile; last access: 05.11.2013 
4 Robert Koch-Institut. Online: http://www.rki.de/Krebs/DE/Content/ ZfKD/Aktuelles/Brustkrebsmortalitaet.html; last access: 02.03.2015

5 Scharl A. Mammographiescreening. Gynäkologe 2014; 5: 325-333

6 Scharl A, Costa SD. Diagnostik und Therapie des duktalen Carcinoma in situ. Gynäkol Prax 2004; 28: 61-79

7 Liedtke C, Thill M, Hanf V et al. AGO recommendations for the diagnosis and treatment of patients with early breast cancer: update 2014 . Breast Care (Basel) 2014; 9: 189-200

8 AGO Kommission Mamma. Diagnostik und Therapie von Patientinnen mit primärem und metastasierten Brustkrebs. Online: http://www. ago-online.de/de/infothek-fuer-aerzte/leitlinienempfehlungen/ mamma/; last access: 02.03.2015

9 Dunne C, Burke JP, Morrow M et al. Effect of margin status on local recurrence after breast conservation and radiation therapy for ductal carcinoma in situ. J Clin Oncol 2009; 27: 1615-1620

10 Onkologisches Leitlinienprogramm. Interdisziplinäre S3-Leitlinie für die Diagnostik, Therapie und Nachsorge des Mammakarzinoms. 2012. Online: http://leitlinienprogramm-onkologie.de/Mammakarzinom.67. 0.html; last access: 02.03.2015

11 Kurian AW, Lichtensztajn DY, Keegan TH et al. Use of and mortality after bilateral mastectomy compared with other surgical treatments for breast cancer in California, 1998-2011. JAMA 2014; 312: 902-914

12 Garcia-Etienne CA, Tomatis M, Heil J et al.; eusomaDB Working Group. Mastectomy trends for early-stage breast cancer: a report from the EUSOMA multi-institutional European database. Eur J Cancer 2012; 48: 1947-1956

13 Pilewskie $M$, Ho A, Orell $E$ et al. Effect of margin width on local recurrence in triple-negative breast cancer patients treated with breastconserving therapy. Ann Surg Oncol 2014; 21: 1209-1214

14 Moran MS, Schnitt SJ, Giuliano AE et al. Society of Surgical OncologyAmerican Society for Radiation Oncology consensus guideline on margins for breast-conserving surgery with whole-breast irradiation in stages I and II invasive breast cancer. J Clin Oncol 2014; 32: 1507-1515

15 Lostumbo L, Carbine NE, Wallace J. Prophylactic mastectomy for the prevention of breast cancer. Cochrane Database Syst Rev 2010; 11: CD002748

16 Scharl A, Salterberg A. Multimodale Therapie des nicht metastasierten Mammakarzinoms. Onkologe 2014; 4: 379-394

17 Houssami N, Turner R, Morrow M. Preoperative magnetic resonance imaging in breast cancer. Meta-analysis of surgical outcomes. Ann Surg 2013; 257: 249-255

18 Houssami N, Turner $R$, Macaskill $P$ et al. An individual person data meta-analysis of preoperative magnetic resonance imaging and breast cancer recurrence. J Clin Oncol 2014; 32: 392-401

19 Brennan ME, Houssami N. Evaluation of the evidence on staging imaging for detection of asymptomatic distant metastases in newly diagnosed breast cancer. Breast 2012; 21: 112-123

20 Gerber B, Seitz E, Müller $\mathrm{H}$ et al. Perioperative screening for metastatic disease is not indicated in patients with primary breast cancer and no clinical signs of tumor spread. Breast Cancer Res Treat 2003; 82: 29-37

21 Schnipper LE, Smith TJ, Raghavan D et al. American Society of Clinical Oncology identifies five key opportunities to improve care and reduce costs: the top five list for oncology. J Clin Oncol 2012; 30: 1715-1724

22 Simos D, Hutton B, Clemons M. Are physicians choosing wisely when imaging for distant metastases in women with operable breast cancer? J Oncol Pract 2014; pii: JOP.2014.000125 [Epub ahead of print]

23 Davies C, Godwin J, Gray R et al.; Early Breast Cancer Trialists' Collaborative Group (EBCTCG). Relevance of breast cancer hormone receptors and other factors to the efficacy of adjuvant tamoxifen: patient-level meta-analysis of randomised trials. Lancet 2011; 378: 771-784

24 Hadji P, Ziller V, Kyvernitakis J et al. Persistence in patients with breast cancer treated with tamoxifen or aromatase inhibitors: a retrospective database analysis. Breast Cancer Res Treat 2013; 138: 185-191

25 Hershman DL, Shao T, Kushi LH et al. Early discontinuation and non-adherence to adjuvant hormonal therapy are associated with increased mortality in women with breast cancer. Breast Cancer Res Treat 2011; 126: 529-537

26 Kidwell KM, Harte SE, Hayes DF et al. Patient-reported symptoms and discontinuation of adjuvant aromatase inhibitor therapy. Cancer 2014; 120: 2403-2411

27 Irwin ML, Cartmel B, Gross CP et al. Randomized exercise trial of aromatase inhibitor-induced arthralgia in breast cancer survivors. J Clin Oncol 2014; pii: JCO.2014.57.1547 [Epub ahead of print]
28 Fellowes D, Fallowfield LJ, Saunders CM et al. Tolerability of hormone therapies for breast cancer: how informative are documented symptom profiles in medical notes for 'well-tolerated' treatments? Breast Cancer Res Treat 2001; 66: 73-81

29 Cuzick J, Sestak I, Forbes JF et al. Anastrozole for prevention of breast cancer in high-risk postmenopausal women (IBIS-II): an international, double-blind, randomised placebo-controlled trial. Lancet 2014; 383 : 1041-1048

30 Francis PA, Regan MM, Fleming GF et al. Adjuvant ovarian suppression in premenopausal breast cancer. N Engl J Med 2015; 372: 436-446

31 Pagani 0, Regan MM, Walley BA et al. Adjuvant exemestane with ovarian suppression in premenopausal breast cancer. N Engl J Med 2014; 371: 107-118

32 Peto R, Davies C, Godwin J et al.; Early Breast Cancer Trialists' Collaborative Group (EBCTCG). Comparisons between different polychemotherapy regimens for early breast cancer: meta-analyses of long-term outcome among 100,000 women in 123 randomised trials. Lancet 2012; 379: 432-444

33 Tolaney SM, Barry WT, Dang CT et al. Adjuvant paclitaxel and trastuzumab for node-negative, HER2-positive breast cancer. N Engl J Med 2015; 372: 134-141

34 Denkert C, von Minckwitz G, Brase JC et al. Tumor-infiltrating lymphocytes and response to neoadjuvant chemotherapy with or without carboplatin in human epidermal growth factor receptor 2-positive and triple-negative primary breast cancers. J Clin Oncol 2014; pii: JCO.2014.58.1967 [Epub ahead of print]

35 von Minckwitz G, Hahnen E, Fasching PA et al. Pathological complete response ( $\mathrm{pCR}$ ) rates after carboplatin-containing neoadjuvant chemotherapy in patients with germline BRCA (gBRCA) mutation and triplenegative breast cancer (TNBC): Results from GeparSixto. J Clin Oncol 2014; 32: 5s (Suppl.; Abstr. 1005)

36 Correa C, McGale P, Taylor C et al.; Early Breast Cancer Trialists' Collaborative Group (EBCTCG). Overview of the randomized trials of radiotherapy in ductal carcinoma in situ of the breast. J Natl Cancer Inst Monogr 2010; 2010: 162-177

37 Wapnir IL, Dignam JJ, Fisher B et al. Long-term outcomes of invasive ipsilateral breast tumor recurrences after lumpectomy in NSABP B-17 and B-24 randomized clinical trials for DCIS. J Natl Cancer Inst 2011; 103: $478-488$

38 Deutsches Mammographiescreeningprogramm. Evaluationsbericht 2011. Online: http://fachservice.mammo-programm.de/download/ Evaluationsbericht_2011_Zusammenfassung_der_Ergebnisse.pdf; last access: 03.04.2015

39 Darby S, McGale P, Correa C et al.; Early Breast Cancer Trialists' Collaborative Group (EBCTCG). Effect of radiotherapy after breast-conserving surgery on 10-year recurrence and 15-year breast cancer death: meta-analysis of individual patient data for 10,801 women in 17 randomised trials. Lancet 2011; 378: 1707-1716

40 Schonberg MA, Marcantonio ER, Ngo L et al. Causes of death and relative survival of older women after a breast cancer diagnosis. J Clin Oncol 2011; 29: 1570-1577

41 Hughes KS, Schnaper LA, Bellon JR et al. Lumpectomy plus tamoxifen with or without irradiation in women age 70 years or older with early breast cancer: long-term follow-up of CALGB 9343. J Clin Oncol 2013; 31: 2382-2387

42 Kunkler IH, Williams LJ, Jack WJ et al. Breast-conserving surgery with or without irradiation in women aged 65 years or older with early breast cancer (PRIME II): a randomised controlled trial. Lancet Oncol 2015; 16: $266-273$

43 von Minckwitz G, Reimer T, Potenberg J et al. The phase III ICE study: adjuvant Ibandronate with or without capecitabine in elderly patients with moderate or high risk early breast cancer. San Antonio Breast Cancer Symposium 2014; Abstr. S3-04

44 Online: https://www.adjuvantonline.com; last access: 02.03.2015

45 Diel IJ, Solomayer EF, Costa SD et al. Reduction in new metastases in breast cancer with adjuvant clodronate treatment. N Engl J Med 1998; 339: 357-363

46 Coleman R, Gnant M, Paterson A et al. Effects of bisphosphonate treatment on recurrence and cause-specific mortality in women with early breast cancer: a meta-analysis of individual patient data from randomized trials. San Antonio Breast Cancer Symposium 2013; Abstr. S4-07

47 Rack B, Andergassen U, Neugebauer J et al. The German SUCCESS C Study - The first European lifestyle study on breast cancer. Breast Care (Basel) 2010; 5: 395-400 
48 Goodwin PJ. Obesity and endocrine therapy: host factors and breast cancer outcome. Breast 2013; 22 (Suppl. 2): S44-S47

49 Chlebowski RT. Nutrition and physical activity influence on breast cancer incidence and outcome. Breast 2013; 22 (Suppl. 2): S30-S37
50 Chlebowski RT, Blackburn GL, Thomson GA et al. Dietary fat reduction and breast cancer outcome: interim efficacy results from the Women's Intervention Nutrition Study (WINS). J Natl Cancer Inst 2006; 98: 1767-1776

51 Chlebowski RT, Blackburn GL. Final survival analysis from the randomized Women's Intervention Nutrition Study (WINS) evaluating dietary intervention as adjuvant breast cancer therapy. San Antonio Breast Cancer Symposium 2014; Abstr. S5-08 